\title{
La Relación de Largo Plazo del PIB Mexicano y de sus Componentes con la Actividad Económica en los Estados Unidos y con el Tipo de Cambio Real
}

\author{
Daniel G. Garcés Díaz \\ dgarces@banxico.org.mx
}

Marzo de 2003

Documento de Investigación No. 2003-4

Dirección General de Investigación Económica

BANCO DE MÉXICO

\footnotetext{
*Agradezco a Rafael Gómez-Tagle, Alejandro Werner, Armando Baqueiro y los participantes en seminarios en el Banco de México, la Universidad de California en Berkeley y el Encuentro Europeo de la Sociedad Econométrica del 2002 en Venecia por sus útiles comentarios y sugerencias. Rocío Elizondo colaboró de modo eficiente en todos los aspectos de este documento. El autor es por supuesto responsable de cualquier error u omisión. Asimismo, el contenido de este trabajo no refleja necesariamente las opiniones del Banco de México.
} 


\title{
La Relación de Largo Plazo del PIB Mexicano y de sus Componentes con la Actividad Económica en los Estados Unidos y con el Tipo de Cambio Real
}

\author{
Daniel G. Garcés Díaz
}

\author{
Marzo de 2003 \\ Documento de Investigación No. 2003-4 \\ Dirección General de Investigación Económica \\ BANCO DE MÉXICO
}

\section{RESUMEN}

En este artículo se investiga la influencia que la integración económica con los Estados Unidos ha tenido sobre el PIB mexicano durante el período 1980-2000. El análisis se basa en la estimación de relaciones de equilibrio de largo plazo y en los respectivos procesos de ajuste para el PIB mexicano y cada uno de sus componentes con el índice de la producción industrial de los Estados Unidos y el tipo de cambio real bilateral. Se encuentra que el impacto de la primera de estas variables sobre el PIB es cercano a uno, indicando que la tendencia de ambas economías durante el período de muestra fue similar. Dicho coeficiente varía en tamaño para cada uno de los componentes. El efecto del tipo de cambio real es positivo sobre la balanza comercial pero negativo sobre el resto de la economía. Si bien este último efecto ya había sido reportado por otros autores, en este artículo proporciona un análisis nuevo y más formal del mismo. 


\section{Introducción}

En este artículo se presenta una perspectiva econométrica del sector real de la economía mexicana para el período 1980-2000. Se estudia el comportamiento de algunas variables importantes en el contexto de relaciones de equilibrio de largo plazo. Los resultados ilustran la evolución e intensidad de los lazos entre las economías de México y los Estados Unidos y el papel que el tipo de cambio real ha tenido en la determinación de la actividad económica en México.

En 1982 México abandonó una política de tipo de cambio fijo a favor de esquemas intermedios; sin embargo, éstos no previnieron la ocurrencia de varias devaluaciones drásticas y crisis económicas antes de que un sistema de flotación libre se aplicara a partir de 1995. Este régimen parece estar funcionando aceptablemente y, por ello, las discusiones sobre cuál sistema cambiario es el más recomendable para el país han perdido vigencia.

México experimentó una prolongada recesión durante los ochenta, comúnmente entendida como una secuela de la crisis de la deuda que estalló en 1982. El país tuvo una evolución económica decorosa desde el comienzo de los noventa hasta el final de 1994 cuando volvió a enfrentar una crisis de proporciones mayores. Después de esto, la economía volvió a crecer a tasas altas de 1996 a 2000, ayudada por el notable desempeño de su sector externo. México es actualmente el segundo socio comercial de los Estados Unidos.

La alta volatilidad de los indicadores económicos de México es un reflejo de éstos y otros eventos. Es posible, sin embargo, representar su comportamiento estocástico con modelos lineales de parámetros constantes que implican que esa variabilidad no modifica de modo fundamental las relaciones económicas. En general, los movimientos bruscos de algunas variables reflejan la volatilidad de los factores con los que están relacionadas y no necesariamente inestabilidad de la estructura económica. El principal objetivo de este trabajo es ofrecer una medición del efecto de dos variables importantes para la economía mexicana: la actividad económica de los Estados Unidos y el tipo de cambio real. Esta última variable se determina dentro del sistema dado el entorno externo. No obstante, 
como se muestra más adelante, se puede analizar la trayectoria de otras variables sin modelar explícitamente su comportamiento probabilístico en tanto se cumplan ciertas condiciones.

Los hallazgos principales son los siguientes: la balanza comercial de México responde de manera robusta a variaciones en la economía de los Estados Unidos y en el tipo de cambio real. Sin embargo, la actividad económica global de México se relaciona negativamente con el tipo de cambio real, tal y como ha sido señalado por varios autores. ${ }^{1}$ Se deriva una relación de largo plazo para el PIB mexicano, el índice de la producción industrial de los Estados Unidos y el tipo de cambio real. Normalizando sobre el coeficiente de cointegración del PIB, el coeficiente para la segunda variable es cercano a uno y el correspondiente al tipo de cambio real es negativo. Esto implica que durante el período de muestra la tendencia del PIB mexicano fue determinada por su relación con los Estados Unidos y que el tipo de cambio real se conecta con las fluctuaciones alrededor de la misma. El modelo es estadísticamente satisfactorio y se desempeña aceptablemente fuera de la muestra.

Cada uno de los componentes principales del PIB genera una relación de cointegración similar: los logaritmos de la formación bruta de capital, el consumo privado, el gasto gubernamental, y las exportaciones e importaciones son también funciones del índice de la producción industrial de los Estados Unidos y del tipo de cambio real.

El papel dominante de la actividad económica de los Estados Unidos parece haber comenzado al alterarse la función del gobierno mexicano después de la crisis de la deuda y al intensificarse los esfuerzos de los productores nacionales para tomar ventaja de la cercanía geográfica a un enorme mercado con el potencial de compensar la debilidad de la demanda interna. Por otra parte, si bien el efecto negativo del tipo de cambio real ha sido estudiado por Kamin y Rogers (2000) y otros autores, en este documento se proporciona una nueva perspectiva en la forma de una relación multivariada de cointegración que permite pruebas más rigurosas.

\footnotetext{
${ }^{1}$ Dos artículos recientes que se refieren a lo anterior son Kamin y Rogers (2000) y Bergoeing et al. (2002).
} 
El resto del documento se organiza como sigue: en la sección II se describen los datos y se examina la posible presencia de raíces unitarias; la sección III contiene el análisis preliminar; en la sección IV se presenta el análisis de cointegración para el PIB y sus componentes; en la sección V se ofrece una interpretación de los resultados y en la sección VI las conclusiones.

\section{Descripción de las Series y Análisis de Raíces Unitarias}

Las series en la muestra son de frecuencia trimestral y cubren el periodo 1980-2000. Todas las variables se utilizan en logaritmos naturales y aquéllas que se generan al menos mensualmente fueron incorporadas tomando la última observación disponible en el trimestre. La actividad económica es medida por el producto interno bruto (pib) y sus componentes en pesos constantes: consumo privado (conspri), gasto gubernamental (consgub), formación bruta de capital ( $f k$ ), exportaciones (exp) e importaciones (imp). El nivel de precios se mide con el índice nacional de precios al consumidor $(p)$. Los indicadores de la actividad económica y del nivel de precios en los Estados Unidos son el índice de la producción industrial (ivusa) y el índice de precios al consumidor (peu), respectivamente. El tipo de cambio bilateral México-Estados Unidos $(e)$ se define en términos de pesos por dólar. El tipo de cambio real (tcr) utilizado es igual a $e+p e u-p$.

El Cuadro 1 contiene las pruebas de raíz unitaria para todas las variables. Un asterisco indica significación estadística al 5\%. El proceso para llegar a la especificación de la prueba para cada variable se inició incluyendo cuatro rezagos, una tendencia lineal y una constante; los términos que no fueron significativos fueron suprimidos. En la columna de especificación (“Espec.”) los símbolos T y C indican si una tendencia y/o un intercepto fueron incluidos al final. 
Cuadro 1:

Pruebas de Raíz Unitaria ADF 1980-2000

\begin{tabular}{|c||c|c|c||c|c|c||}
\hline \multicolumn{1}{|c||}{ Variable } & \multicolumn{3}{c||}{ Niveles } & \multicolumn{3}{c||}{ Diferencias } \\
\cline { 2 - 7 } & Rezagos & Espec. $^{\text {a }}$ & Estadístico & Rezagos & Espec. & Estadístico \\
\hline \hline \multirow{2}{*}{ rer } & 0 & $\mathrm{C}$ & -2.14 & 0 & $\mathrm{~N}$ & $-10.10^{*}$ \\
ivusa & 1 & $\mathrm{~T} \& \mathrm{I}$ & -1.97 & 0 & $\mathrm{C}$ & $-7.49^{*}$ \\
gdp & 4 & $\mathrm{~T} \& \mathrm{I}$ & -2.96 & 4 & $\mathrm{C}$ & $-4.01^{*}$ \\
fk & 4 & $\mathrm{~T} \& \mathrm{I}$ & -3.33 & 0 & $\mathrm{C}$ & $-8.10^{*}$ \\
ivpi & 4 & $\mathrm{~T} \& \mathrm{I}$ & -2.88 & 3 & $\mathrm{C}$ & $-3.86^{*}$ \\
consgub & 4 & $\mathrm{~T} \& \mathrm{I}$ & -3.31 & 2 & $\mathrm{C}$ & $-32.35^{*}$ \\
conspri & 4 & $\mathrm{~T} \& \mathrm{I}$ & -2.95 & 4 & $\mathrm{C}$ & $-3.66^{*}$ \\
exp & 4 & $\mathrm{~T} \& \mathrm{I}$ & -1.79 & 4 & $\mathrm{~T} \& \mathrm{I}$ & $-4.96^{*}$ \\
imp & 4 & $\mathrm{~N}$ & 1.02 & 4 & $\mathrm{~N}$ & $-3.27^{*}$ \\
\hline \hline
\end{tabular}

${ }^{\text {a }}$ Especificación Final. T, I y N indican la presencia de tendencia, intercepto y nada, respectivamente.

* Significativo al menos al 5\%

En la mayoría de los casos, los resultados son los mismos si la especificación de la prueba se cambia de modo razonable e indican que todas las variables son I(1), lo cual para algunas de ellas no está exento de controversia. No obstante, los resultados principales del artículo se mantendrían aun si alguna de ellas tuviera un orden de integración menor (como es el posible caso del tipo de cambio real).

\section{Análisis Preliminar}

En esta sección se presentan de manera informal algunos de los argumentos principales del trabajo. En especial, se discuten aspectos del papel que la economía estadounidense y el tipo de cambio real han tenido en México. La economía de los Estados Unidos tiene una importancia evidente para México, pero la obtención de una medición de su impacto pleno puede no ser obvia. Aunque cerca de $90 \%$ de las exportaciones mexicanas se dirige a aquel país y las tasas de crecimiento promedio de ambas economías son muy similares, la correlación entre las tasas trimestrales es relativamente baja. La explicación de ello es la presencia de un elemento altamente volátil en el PIB mexicano que no está relacionado con la actividad económica de los Estados Unidos. ${ }^{2}$

\footnotetext{
${ }^{2}$ Por ejemplo, la correlación entre las tasa de crecimiento del PIB mexicano y la del índice de la producción industrial de los Estados Unidos para el período 1980-2000 es marginalmente significativa. Sin embargo, en los últimos años la relación entre ambas series se ha vuelto más evidente debido a la menor volatilidad del tipo de cambio real.
} 
Este componente de alta variabilidad está relacionado con el tipo de cambio real de una manera muy estrecha. En la Gráfica 1 se proporciona una primera aproximación a esta relación. En ella se muestra a la desviación del logaritmo desestacionalizado del PIB mexicano respecto de su tendencia, obtenida con el filtro HP, y con relación al negativo del tipo de cambio real (-tcr); en dicha gráfica un movimiento hacia arriba indica apreciación. Obsérvese que los movimientos del tipo de cambio real preceden a los del PIB sin tendencia, aunque debe enfatizarse que la gráfica por si misma no puede considerarse una prueba formal de causalidad.

\section{Gráfica 1}

\section{Relación Entre el PIB sin Tendencia (pib) y el Tipo de Cambio Real (tcr)}

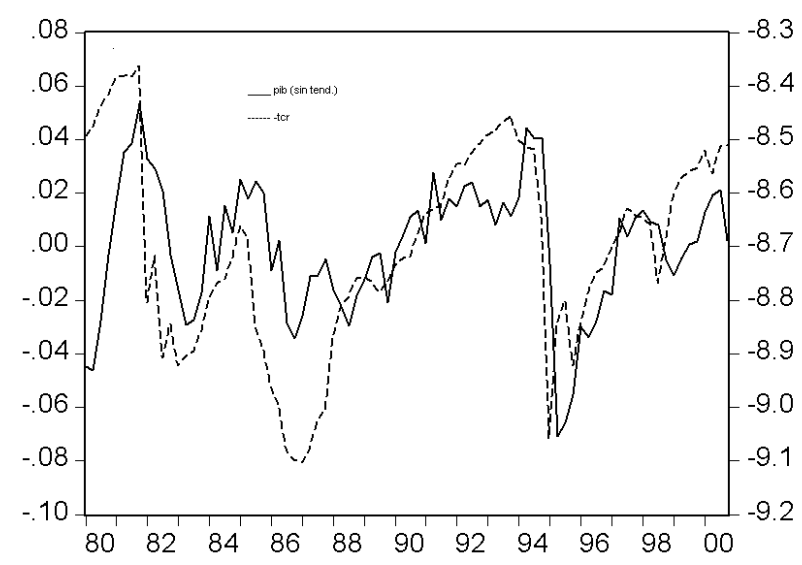

La similitud de las trayectorias de ambas series es evidente. Una importante característica de esta relación es que una apreciación está relacionada con una desviación de tendencia positiva y viceversa. Esto último, contrario a lo que muchos modelos económicos predicen. Este efecto ha sido ampliamente estudiado (ver Kamin y Rogers, 2000 para una revisión de la literatura).

Para mostrar que este efecto coexiste con un estímulo positivo (negativo) de una depreciación (apreciación) del tipo de cambio real sobre el superávit (déficit) comercial, se muestran las funciones ortogonales de impulso respuesta de un $\operatorname{VAR}(4)$ no restringido con los niveles del tipo de cambio real (tcr), el logaritmo del cociente de exportaciones a importaciones (exp-imp) y el logaritmo del PIB mexicano (pib). Por simplicidad se deja fuera a la actividad económica de los Estados Unidos puesto que su inclusión, o el orden de las variables, no cambia los aspectos cualitativos que se quieren mostrar aquí. Los 
renglones muestran la respuesta de una variable dada a perturbaciones de cada variable del sistema. La columna de gráficas indica el efecto que una perturbación dada tiene sobre cada una de las variables del sistema.

\section{Gráfica 2}

Efectos de Perturbaciones Sobre el Tipo de Cambio Real, la Balanza Comercial y el PIB.

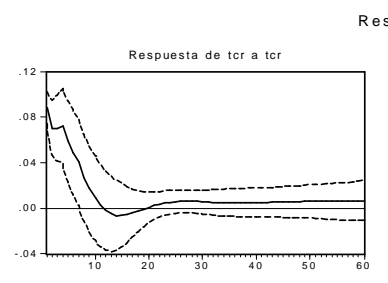

Respuesta Cholesky a Inovaciones de Una D.E. \pm 2 S.E.
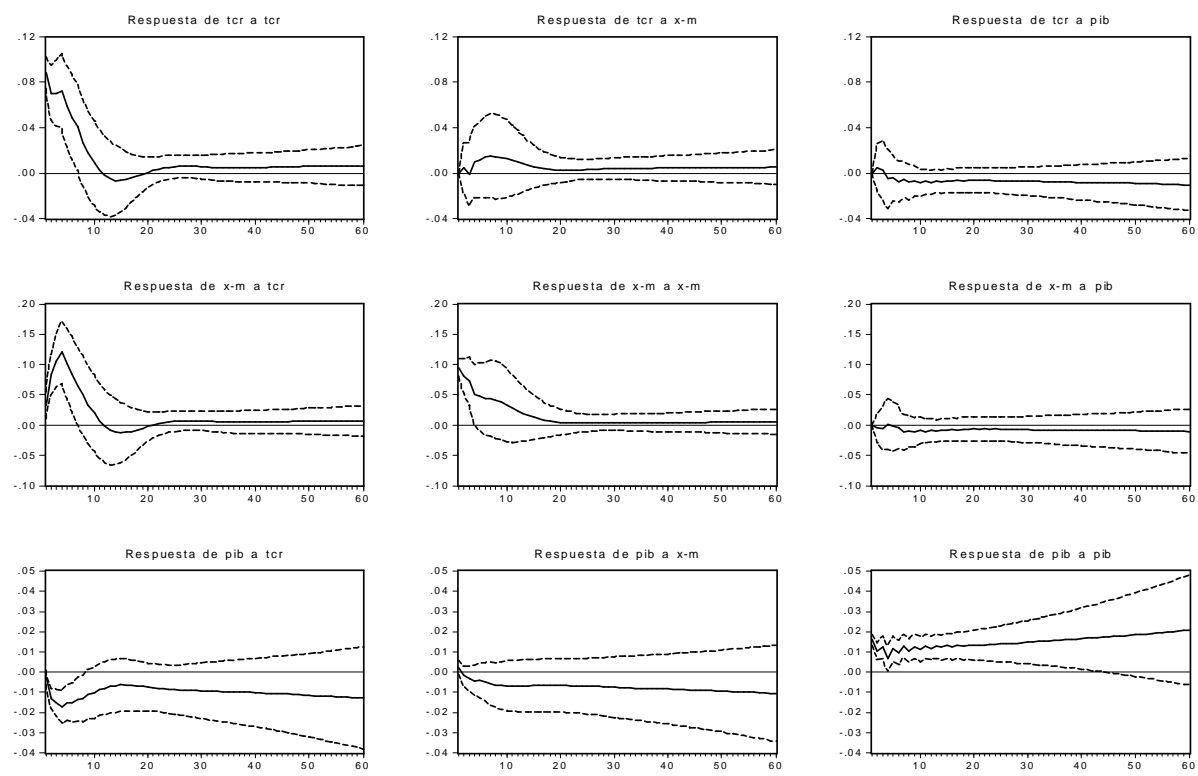

Perturbaciones de la balanza comercial (segunda columna) y del PIB (tercera columna) no tienen un efecto significativo sobre el tipo de cambio real. Por otra parte, una perturbación (depreciación) del tipo de cambio real tiene un fuerte impacto sobre las otras dos variables. Sin embargo, el signo del efecto es diferente: es positivo sobre la balanza comercial pero negativo sobre el PIB. Kamin y Rogers (2000) demuestran que el efecto contraccionista de una devaluación sobre el PIB es robusto en los VAR con diferentes variables que ellos analizan, aunque éstos no incluyen a la balanza comercial. En la siguiente sección se propone un nuevo enfoque para analizar simultáneamente los efectos de la demanda externa y del tipo de cambio real sobre el PIB mexicano y sus componentes.

\section{Análisis de las Relaciones de Largo Plazo}

En esta sección se aplica un análisis de cointegración para estudiar las relaciones de algunas variables macroeconómicas de México con la actividad económica en los Estados Unidos y con el tipo de cambio real. Para el PIB se aplican tanto el método multivariado de 
Johansen como el del modelo de corrección de error no restringido para obtener los parámetros de largo plazo. Para el resto de las variables solamente se utiliza el segundo procedimiento.

\section{IV.1 Efectos de la Demanda Externa y del Tipo de Cambio Real sobre el PIB}

El punto de partida es el análisis de la relación entre el PIB mexicano, el índice de la producción industrial de los Estados Unidos y el tipo de cambio real en un marco de cointegración. Se utiliza el índice de producción industrial en lugar del PIB estadounidense debido a que tanto la inferencia estadística como la evaluación fuera de la muestra resultaron mejores con la primera de estas variables. Esto es quizás indicativo de que la economía mexicana es afectada por el sector industrial más directamente que otras ramas de la economía de los Estados Unidos aunque otras interpretaciones son posibles.

Los resultados del análisis con el procedimiento de Johansen se presentan en el Cuadro 2. Se incluyen cuatro variables dicotómicas para asegurar la normalidad de los residuales. La especificación incluye una constante en el espacio de cointegración.

\section{Cuadro 2}

Análisis de Máxima Verosimilitud Para el Log. del PIB 1980-2000

\begin{tabular}{|c|c|c|c|c|}
\hline \multicolumn{5}{|c|}{ (i) Análisis de Cointegración } \\
\hline Eigenvalores & 0.34 & . & 0.13 & 0.05 \\
\hline Hipótesis Nula & $\operatorname{ran} .=0$ & . & $\operatorname{ran} . \leq 1$ & $\operatorname{ran} . \leq 2$ \\
\hline$\lambda$ Estadístico traza & $47.57^{* *}$ & . & 1.54 & 3.99 \\
\hline Valores Críticos (95\%) & 29.70 & . & 15.40 & 3.80 \\
\hline
\end{tabular}

(ii) Vector de Cointegración Estandarizado y Coeficientes de Ajuste

\begin{tabular}{lcccc}
\hline \hline Variable & pib &. & ivusa & Tcr \\
Coef. De Coint. Norm. $\gamma$ & 1.00 &. & -0.69 & 0.33 \\
Coeficiente de Ajuste $\alpha$ & -0.16 &. & 0.13 & -0.42 \\
\hline
\end{tabular}

(iii) Pruebas de Significatividad y Exogeneidad Débil

\begin{tabular}{lcccc}
\hline \hline Variable & Pib & & ivusa & tcr \\
Exclusión $\chi 2(1)$ & $22.02^{* *}$ &. & $21.63^{* *}$ & $27.32^{* *}$ \\
Exogeneidad Débil $\chi 2(1)$ & $9.96^{* *}$ &. & 9.62 & 5.74 \\
\hline \multicolumn{4}{c}{ (vi) Pruebas de Especificación } \\
\hline \hline Ecuación & pib & ivusa & tcr & Pruebas Conjuntas \\
AR F $(5,65)$ & $1.18[.97]$ & $1.49[.21]$ & $0.52[.76]$ & $\mathrm{F}(45,158)=0.911 .49[.63]$ \\
Normalidad $\chi 2(2)$ & $3.05[.22]$ & $1.64[.44]$ & $4.20[.12]$ & $\chi 2(6)=8.76[.63]$ \\
Heteroced. F $(30,25)$ & $0.49[.97]$ & $0.42[.99]$ & $0.93[.58]$ & $\mathrm{F}(162,225)=0.56[.99]$ \\
\hline
\end{tabular}

Los valores $\mathrm{p}$ van entre paréntesis rectangulares.

Ver Hendry (1995) para una descripción detallada de las pruebas 
Los resultados presentados en el cuadro demuestran que hay un solo vector de cointegración y que las tres variables rechazan la hipótesis de exclusión. Ni el índice de la producción industrial de los Estados Unidos (ivusa) ni el tipo de cambio real (tcr) rechazan la hipótesis de exogeneidad débil y, por ello, es posible derivar una ecuación para el PIB condicionanda en las otras dos variables. El signo negativo del coeficiente para el tipo de cambio real proviene de la coincidencia de períodos de apreciación (depreciación) con episodios de expansión (contracción) económica y ha sido estudiado antes por otros autores con diferentes métodos, como ya se mencionó.

La cointegración entre estas tres variables es estable durante el período 1980-2000, tal como se muestra en la Gráfica 2. Esta fue construida al estimar recursivamente un cociente de verosimilitud. Dado que la línea de prueba se encuentra muy por debajo de la línea del valor crítico, la conclusión es que no hubo quiebres estructurales en esta relación.

\section{Gráfica 3}

\section{Prueba de Estabilidad Recursiva para la Ecuación de Largo Plazo del PIB}

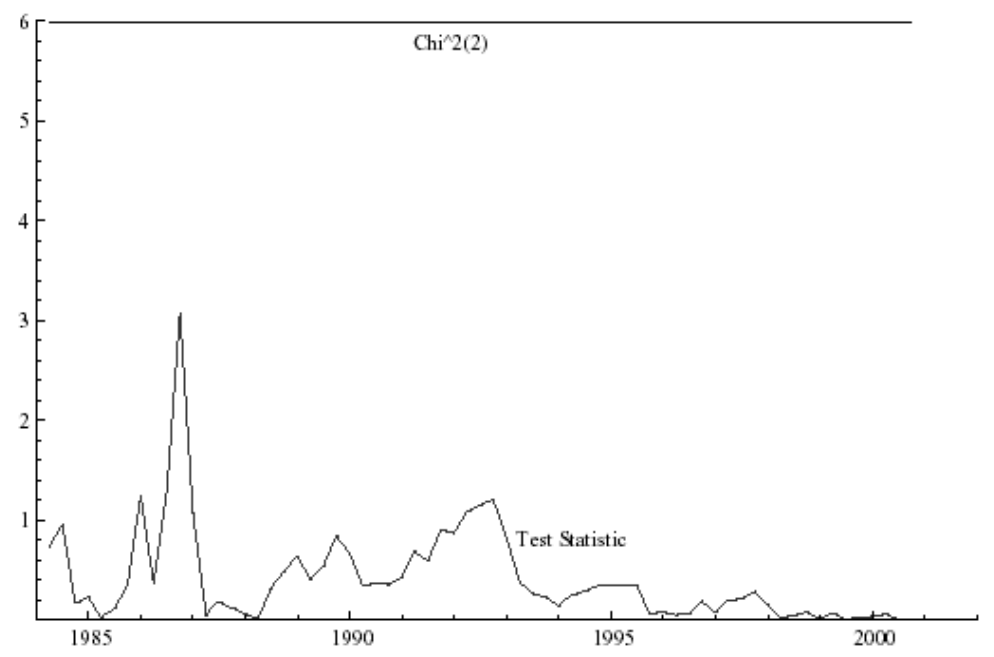

A continuación, un modelo de corrección de error no restringido es estimado para la tasa de crecimiento del PIB mexicano. Los resultados se presentan en el Cuadro 2. El modelo tiene buenas propiedades estadísticas y un notable ajuste. Solamente requirió, además de indicadores estacionales, variables dicotómicas para los dos primeros trimestres de la crisis de 1995 para obtener la normalidad de los residuales. Varios estadísticos de evaluación se incluyen después del renglón que indica el período de estimación. El primer 
estadístico es el valor p para el estadístico ECM (en este caso el cociente t del coeficiente del PIB mexicano rezagado) obtenido del programa de Ericsson y MacKinnon (1999). Los otros estadísticos son los coeficientes de determinación, el error estándar de la regresión, los estadísticos para probar asimetría, curtosis, normalidad, autocorrelación y efectos ARCH hasta el séptimo rezago, el estadístico de White para heterocedasticidad y el estadístico RESET. Dos asteriscos indican significación al 5\%. 


\section{Cuadro 3}

Modelo de Corrección de Error para el PIB 1980-2000

\begin{tabular}{|c|c|}
\hline Variable & $\Delta g d p t$ \\
\hline gdpt-1 & $\begin{array}{l}-0.201 \\
(0.041)\end{array}$ \\
\hline ivusat-1 & $\begin{array}{l}0.167 \\
(0.029) \\
\end{array}$ \\
\hline rert-1 & $\begin{array}{l}-0.066 \\
(0.012)\end{array}$ \\
\hline$\Delta g d p t-1$ & $\begin{array}{l}-0.340 \\
(0.081)\end{array}$ \\
\hline$\Delta g d p t 3$ & $\begin{array}{l}-0.290 \\
(0.076)\end{array}$ \\
\hline$d 9501$ & $\begin{array}{l}-0.046 \\
(0.014)\end{array}$ \\
\hline$d 9502$ & $\begin{array}{l}-0.075 \\
(0.015)\end{array}$ \\
\hline
\end{tabular}

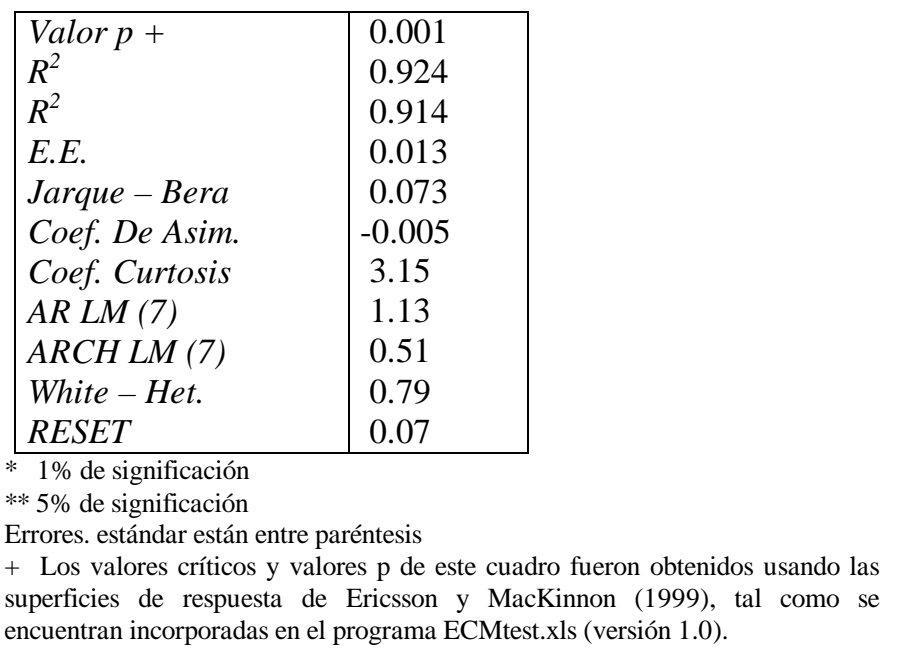

El estadístico ECM confirma la existencia de cointegración a cualquier nivel convencional de significación. Los estadísticos de especificación no detectan problema alguno. El análisis recursivo de esta ecuación, no presentado aquí pero disponible solicitándolo al autor, muestra que el modelo de corrección de error es muy estable y sus parámetros son constantes. Ninguna evidencia de cambio estructural fue encontrada, tal y como ocurría con la relación de largo plazo. La ecuación de largo plazo para el PIB mexicano se obtiene de los coeficientes del cuadro anterior:

$$
p i b=\mathrm{c}+0.83 \text { ivusa }-0.30 \mathrm{tcr}
$$


Esta relación establece que el PIB mexicano en el largo plazo crecerá en cerca de $0.8 \%$ por cada punto que el índice de la producción industrial de los Estados Unidos se incremente. El PIB mexicano decrecerá $0.3 \%$ por cada punto de depreciación del tipo de cambio real. Durante el período de muestra el índice de la producción industrial de los Estados Unidos determinó la tendencia de la economía mexicana y las fluctuaciones del tipo de cambio real empalman muy cercanamente con las desviaciones con respecto a la misma. Esto puede ser apreciado en la Gráfica 3, donde al logaritmo del PIB se le sustrae el logaritmo del índice de la producción industrial estadounidense multiplicado por el valor del coeficiente 0.8 y luego se compara con el negativo del tipo de cambio real.

\section{Gráfica 4}

Representación de la Relación de Largo Plazo Entre el PIB (pib), la Producción Industrial de los Estados Unidos (ivusa) y el Tipo de Cambio Real (tcr).

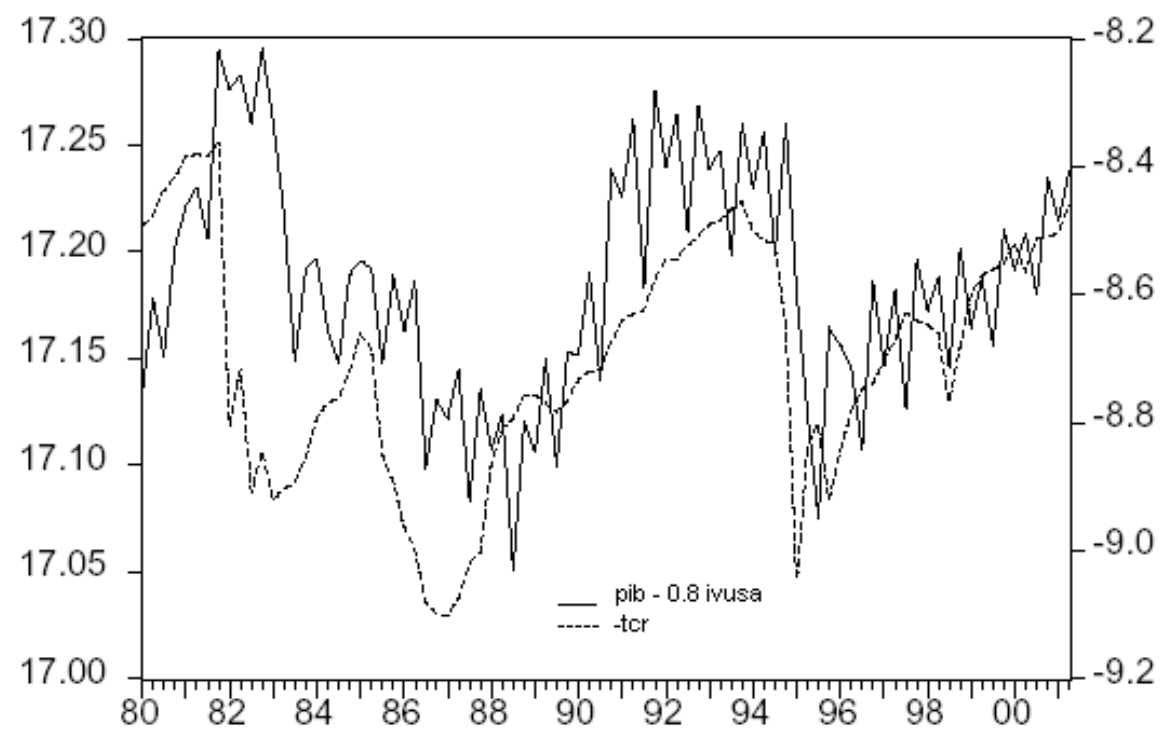

La Gráfica 4 es notablemente similar a la Gráfica 1, donde la tendencia del PIB mexicano fue eliminada con un filtro HP. La implicación es que la tendencia del PIB mexicano fue completamente determinada por la actividad económica de los Estados Unidos. En la sección siguiente se extiende el análisis a los componentes del PIB.

\section{IV.2 Análisis de los Componentes del PIB}

Las variables que se analizan aquí son la formación bruta de capital ( $f k)$, el gasto gubernamental (consgub), el consumo privado (conspri), las exportaciones totales (exp) y 
las importaciones totales (imp). Se muestra que todas ellas dependen del mismo conjunto de variables que el PIB. Se estima un modelo de corrección de error no restringido para cada variable. La prueba de cointegración para cada una hace uso del valor p del estadístico ECM generado por el programa de Ericsson y MacKinnon (1999). La solución de largo plazo para cada modelo proporciona las elasticidades de largo plazo correspondientes (es decir, los vectores de cointegración normalizados).

La dependencia de todos los componentes del PIB mexicano de la actividad económica de los Estados Unidos y del tipo de cambio real es peculiar en sí misma, aunque lo más notable es que tales nexos se puedan describir en el contexto de relaciones de cointegración. El hecho de que el gasto gubernamental responda a las mismas variables que el gasto privado indica su limitado papel en el largo plazo como instrumento de política económica durante el período de la muestra.

El Cuadro 4 contiene los estimados para cada modelo con la excepción de los coeficientes de las variables estacionales. Hay dos ecuaciones para exportaciones y cada una cubre una mitad de la muestra por razones que serán discutidas más adelante. La variable genérica $E C^{\mathrm{i}}$ es el término de corrección de error con $i=f k$, congub, conspri, exp, imp indicando al componente que refiere. Esta se forma factorizando los parámetros de largo plazo estimados en cada ecuación de tal modo que su estadístico t puede ser usado directamente para una prueba de cointegración. Cada término de corrección de error se define al final del cuadro. Los errores estándar están entre paréntesis. 


\section{Cuadro 4}

Modelos de Corrección de Error para los Componentes del PIB

\begin{tabular}{|c|c|c|c|c|c|c|}
\hline Variables & $\Delta \mathrm{fk}_{\mathrm{t}}$ & $\Delta$ consgubt & $\Delta$ conspri $i_{t}$ & $\Delta \exp 80_{t}{ }^{a}$ & $\Delta \exp 90_{t}$ & $\Delta \mathrm{imp}_{\mathrm{t}}$ \\
\hline$E C_{t-1}^{i}$ & $\begin{array}{c}-0.287 \\
(0.056)\end{array}$ & $\begin{array}{l}-0.595 \\
(0.104)\end{array}$ & $\begin{array}{c}-0.247 \\
(0.038)\end{array}$ & $\begin{array}{c}-0.351 \\
(0.089)\end{array}$ & $\begin{array}{l}-0.480 \\
(0.077)\end{array}$ & $\begin{array}{l}-0.250 \\
(0.048)\end{array}$ \\
\hline$\Delta$ consgubt-4 & & $\begin{array}{c}0.210 \\
(0.084)\end{array}$ & & & & \\
\hline$\Delta$ consprit-3 & & & $\begin{array}{c}-0.314 \\
(0.091)\end{array}$ & & & \\
\hline$\Delta \mathrm{m}_{\mathrm{t}-1}$ & & & & & & $\begin{array}{c}0.223 \\
(0.099)\end{array}$ \\
\hline d8103 & & & & $\begin{array}{l}-0.209 \\
(0.063)\end{array}$ & & \\
\hline 8601 & & & & $\begin{array}{c}-0.171 \\
(0.061)\end{array}$ & & \\
\hline d9002 & & & & & $\begin{array}{c}-0.359 \\
(0.037)\end{array}$ & \\
\hline $\mathrm{d} 9501_{\mathrm{t}}$ & $\begin{array}{c}-0.267 \\
(0.041)\end{array}$ & & & & & \\
\hline $\mathrm{d} 9502_{t}$ & $\begin{array}{l}-0.143 \\
(0.041) \\
\end{array}$ & & & & & \\
\hline$T$ & $83-2000$ & $83-2000$ & $81-2000$ & $80-89$ & $90-2000$ & $80-2000$ \\
\hline $\begin{array}{l}\text { ECM p_val }{ }^{+} \\
\mathrm{R}^{2} \\
\mathrm{R}_{\text {adjust }}^{2} \\
\mathrm{SE} \\
\text { Skewness } \\
\text { Kurtosis } \\
\text { Jarque-Bera } \\
\mathrm{LM}_{7} \mathrm{AR} \\
\mathrm{LM} \text { ARCH } \\
\text { White-Het } \\
\text { Reset } \\
\end{array}$ & $\begin{array}{c}0.001 \\
0.694 \\
0.665 \\
0.390 \\
0.13 \\
2.972 \\
0.206 \\
0.994 \\
0.825 \\
0.537 \\
0.011 \\
\end{array}$ & $\begin{array}{l}0.000 \\
0.983 \\
0.981 \\
0.035 \\
0.322 \\
3.216 \\
1.384 \\
1.109 \\
0.852 \\
0.673 \\
0.468 \\
\end{array}$ & $\begin{array}{c}0.000 \\
0.822 \\
0.810 \\
0.020 \\
-0.261 \\
3.538 \\
1.876 \\
1.766 \\
2.396^{\star \star} \\
1.461 \\
0.015 \\
\end{array}$ & $\begin{array}{l}0.046 \\
0.603 \\
0.497 \\
0.057 \\
0.036 \\
1.993 \\
1.656 \\
0.294 \\
0.578 \\
1.483 \\
0.047 \\
\end{array}$ & $\begin{array}{l}0.000 \\
0.802 \\
0.763 \\
0.034 \\
0.041 \\
2.397 \\
0.679 \\
0.274 \\
0.844 \\
1.805 \\
0.926 \\
\end{array}$ & $\begin{array}{c}0.002 \\
0.912 \\
0.941 \\
0.017 \\
0.224 \\
3.424 \\
1.283 \\
1.388 \\
2.097^{\star} \\
1.557^{\star} \\
1.807 \\
\end{array}$ \\
\hline $\begin{array}{lll}\text { *** } & 1 \% & \text { significaci } \\
\text { ** } & 5 \% & \text { significac } \\
* & 10 \% & \text { significac }\end{array}$ & & & & & & \\
\hline \multicolumn{7}{|c|}{$\mathrm{CE}^{\mathrm{fk}}=\mathrm{fk}-1.06$ ivusa $+0.71 \mathrm{tcr}$} \\
\hline \multicolumn{7}{|c|}{$\mathrm{CE}^{\text {consgu }}=$ consgub -0.43 ivusa $+0.16 \mathrm{tcr}$} \\
\hline \multicolumn{7}{|c|}{$\mathrm{CE}^{\mathrm{conspr}}=$ conspri -0.79 ivusa $+0.36 \mathrm{tcr}$} \\
\hline \multicolumn{7}{|c|}{$C E^{\times 8089}=x-1.05$ ivusa $-0.54 \mathrm{tcr}$} \\
\hline \multicolumn{7}{|c|}{$C E^{x 9000}=x-3.04$ ivusa $-0.34 \mathrm{tcr}$} \\
\hline \multicolumn{7}{|c|}{$C E^{m}=m-3.13 i v p i+1.23 \mathrm{tcr}$} \\
\hline
\end{tabular}

Todas las ecuaciones son parsimoniosas, requieren pocos o ningún indicador de valores extremos y exhiben un buen comportamiento estadístico. Hay muy pocos rezagos, lo que indica que el comportamiento dinámico proviene de los términos de corrección de error. 
Los coeficientes para estos últimos, llamados velocidad de ajuste, poseen el signo correcto y van desde -0.25 para la inversión bruta hasta -0.59 para el gasto gubernamental. Las velocidades de ajuste de la formación bruta de capital, consumo privado e importaciones son muy similares entre sí. Las velocidades de ajuste para las exportaciones son más altas, especialmente para el segundo período.

Los valores $\mathrm{p}$ de los estadísticos ECM muestran que hay cointegración con un nivel de significación de 1\%, con la excepción de las exportaciones para el período 1980-89, no obstante que aún en este caso hay cointegración al nivel de 5\%. Los coeficientes de determinación son altos en todos los casos, especialmente para la ecuación de gasto gubernamental (esto obedece a la elevada estacionalidad de este componente). No hay problemas de no normalidad para los residuales en ningún caso, de acuerdo a los estadísticos Jarque-Bera. El estadístico del multiplicador de Lagrange para autocorrelación hasta orden 7 no detecta este problema. Las ecuaciones de consumo privado e importaciones presentan alguna evidencia de efectos $\mathrm{ARCH}$, mientras que la segunda de ellas también muestra problemas de heterocedasticidad de acuerdo a la prueba de White. El estadístico RESET sugiere en cada instancia que la especificación lineal es la apropiada. Las relaciones de largo plazo que implican estos resultados son las siguientes:

$$
\begin{aligned}
f k & =1.06 \text { ivusa }-0.71 \mathrm{tcr} \\
\text { consgov } & =0.43 \text { ivusa }-0.16 \mathrm{tcr} \\
\text { conspri } & =0.79 \text { ivusa }-0.36 \mathrm{tcr} \\
\text { exp } 80 & =1.05 \text { ivusa }+0.54 \mathrm{tcr} \\
\text { exp90 } & =3.04 \text { ivusa }+0.34 \mathrm{tcr} \\
\text { imp } & =3.13 \text { ivusa }-1.23 \mathrm{tcr}
\end{aligned}
$$

Obsérvese que en casi todos los componentes del PIB el efecto del tipo de cambio real es negativo con la excepción de las exportaciones donde el signo es positivo. Esto es congruente con los resultados del análisis de impulso-respuesta del VAR mostrado anteriormente: una depreciación real mejora la balanza comercial pero, debido a la respuesta de los otros componentes, el efecto total resulta contraccionista. 
Los coeficientes para las ecuaciones de exportación son muy diferentes unos de otros, especialmente los correspondientes al índice de la producción industrial de los Estados Unidos. Este coeficiente pasó de alrededor de uno en la primera submuestra cerca de tres en la segunda. La dificultad para encontrar una relación de cointegración en el componente de exportaciones para la muestra completa es probablemente debida a un cambio estructural en los ochenta, cuando México abrió su economía y se adhirió al GATT. Este cambio, sin embargo, no fue tan conspicuo como para reflejarse en la ecuación de PIB.

Las ecuaciones estáticas, o de largo plazo, del 2 al 7, reproducen el comportamiento de los datos bastante bien, aún sin incluir la dinámica de corto plazo. Las cinco secciones de la Gráfica 4 muestran la senda temporal de cada variable junto con el ajuste de largo plazo correspondiente. La gráfica para las exportaciones utilizó las ecuaciones de cada submuestra. 


\section{Gráfica 5}

\section{Bondad del Ajuste de las Ecuaciones de Largo Plazo}
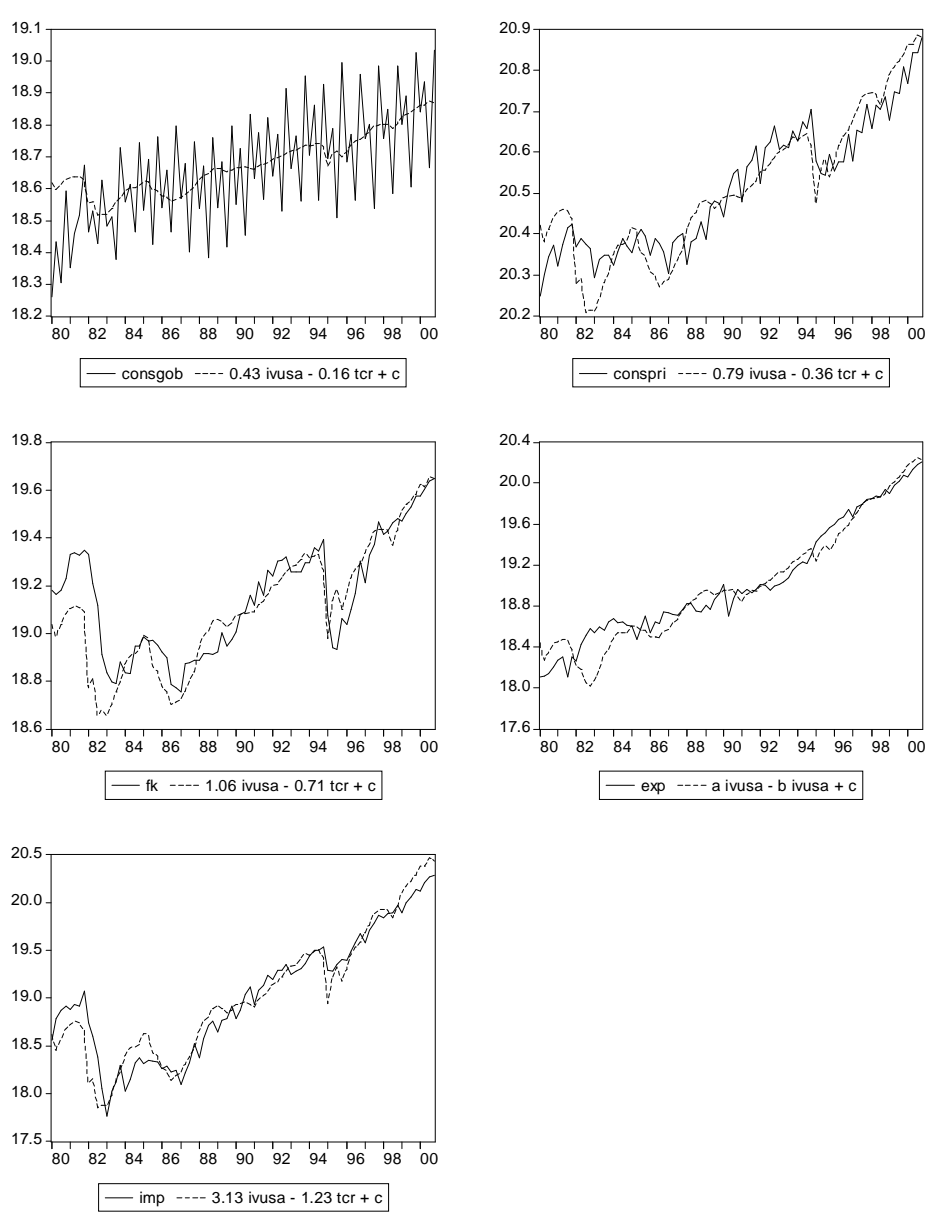

En todos los casos las ecuaciones estáticas reproducen adecuadamente el comportamiento de largo plazo de las variables respectivas, incluyendo muchos cambios de dirección abruptos. La serie de tiempo del gasto gubernamental (segundo recuadro superior) muestra una elevada estacionalidad, la que se captura con variables dicotómicas en el modelo de corto plazo correspondiente. En el mismo recuadro se puede ver que el ajuste antes de 1983 no es tan aceptable porque el papel del gobierno se modifica después de la crisis de la deuda.

\section{Interpretación de los Resultados}

Los resultados de este artículo requieren una discusión más amplia. A lo largo de la muestra el PIB mexicano y sus componentes fueron determinados fundamentalmente por el índice 
de la producción industrial de los Estados Unidos y por el tipo de cambio real. El signo del efecto de esta variable sobre el PIB es negativo. El efecto contraccionista de una devaluación había sido observado en México durante la crisis cambiaria de 1976-77, pero el grado de integración con la economía estadounidense es algo propio del período de muestra. En efecto, la economía mexicana antes de los ochenta solía crecer más rápido que la de los Estados Unidos. Hay varios aspectos que deben ser comentados.

\section{V.1 Discusión Adicional Sobre el Enfoque Econométrico}

El enfoque metodológico seguido para derivar las ecuaciones hace improbable que los resultados puedan ser refutados introduciendo otras variables o utilizando otros métodos. Las relaciones econométricas fueron obtenidas siguiendo un enfoque de lo general a lo específico con detallada comprobación de la especificación y evaluación fuera de la muestra. Estos pasos son parte de lo que Hoover y Perez (1999) y Campos y Ericsson (2000) denominan "minería de datos constructiva". El hecho de que estas ecuaciones fueran tan robustas de acuerdo a varias pruebas rigurosas hace casi imposible que relaciones falaces pudieran producir tales resultados persistentemente. ${ }^{3}$

El papel del tipo de cambio real es especialmente intrigante porque una depreciación está asociada con una contracción del PIB a pesar de tener un efecto positivo sobre el superávit comercial. Esta relación no es en modo alguno nueva en la literatura. La novedad del hallazgo de este artículo es la presencia de tal efecto en el contexto de una relación lineal de largo plazo. ${ }^{4}$ Esto implica que la relación entre las fluctuaciones del PIB y las del tipo de cambio no varía ni con la magnitud ni con el signo de los movimientos de éste. Así una depreciación (apreciación) está asociada con una contracción (expansión) del producto.

Más aún, el resultado es invariante en tanto que se sustenta en una relación de cointegración y no depende de la posible ausencia de otras variables que pudieran ser relevantes. En

\footnotetext{
${ }^{3}$ Que el PIB de un país pueda tener inesperadas relaciones de cointegración se ejemplifica también en Ericsson et al. (2001). Estos autores muestran que el PIB del G7 está cointegrado con la respectiva inflación, resultado que contradice al típico análisis en la literatura sobre crecimiento, donde la inflación a menudo se presume que tiene un efecto negativo sobre la tasa de crecimiento del PIB.

${ }^{4}$ Ninguna ecuación muestra signos de no linealidad. El estadístico RESET reportado en los cuadros puede usarse como una simple prueba de no linealidad, tal y como se discute en Granger y Teräsvirta (1993). El bajo valor de dicho estadístico indica que una especificación lineal es apropiada en cada caso.
} 
efecto, ecuaciones de cointegración como las obtenidas en este documento sobreviven aun si otras variables son necesarias para describir mejor una economía. Ello, porque un sistema con variables adicionales relevantes podría generar otras relaciones de este tipo pero no modificaría las que ya se encontraron.

Sin embargo, las propiedades dinámicas del sistema tales como la de exogeneidad débil de algunas variables, si podrían verse afectadas. ${ }^{5}$ Esto es importante principalmente para el tipo de cambio real puesto que podría ser posible que la introducción de nuevas variables lo hicieran débilmente endógeno aun cuando las relaciones de cointegración sobrevivan. No obstante, resulta muy difícil encontrar variables que puedan causar este cambio en la dinámica del sistema, tal y como Kamin y Rogers (2000) lo señalaron. La razón principal es que el tipo de cambio real tiene las propiedades de una variable financiera $\mathrm{y}$, en consecuencia, una propensión marcada a mostrarse como variable exógena en el análisis estadístico.

Bergoeing, Kehoe, Kehoe y Soto (2001a) invocan el efecto contraccionista de una devaluación para apoyar algunos de sus argumentos pero no lo incorporan ni en sus cálculos ni en sus esfuerzos de modelación. Kamin y Rogers (2000) revisan la literatura sobre dicho efecto $\mathrm{y}$ analizan las funciones de impulso respuesta de vectores autorregresivos con diferentes variables para eliminar la posibilidad de causalidad inversa, correlación espuria y efectos temporales. Los mismos tópicos se pueden examinar en el contexto de cointegración que aquí se proporciona. Los posibles problemas de correlación espuria y efectos temporales son directamente eliminados con las pruebas de cointegración presentadas, tal como se discutió antes, y sólo resta el de causalidad inversa. Esta última posibilidad puede ser descartada probando que el tipo de cambio real es superexógeno con respecto a los parámetros de la ecuación de corrección de error, lo cual se hace aquí aplicando la prueba (i) discutida en Ericsson e Irons (1995). ${ }^{6}$

Nótese que: a) el tipo de cambio real es débilmente exógeno con respecto a los parámetros de la ecuación para el PIB, de acuerdo al Cuadro 2; b) Las pruebas recursivas (no

\footnotetext{
${ }^{5}$ Ver Juselius (2001).

${ }^{6} \mathrm{La}$ imposibilidad de invertir una ecuación en presencia de la propiedad de superxogeneidad es discutida en Hendry y Ericsson (1991).
} 
mostradas aquí pero disponibles requiriéndolas al autor) muestran que el modelo del Cuadro 3 es constante durante el período de muestra; y c) las pruebas recursivas de un modelo autorregresivo para el tipo de cambio real muestran que los cambios de política (por ejemplo, la sustitución de un régimen de tipo de cambio por otro) causaron quiebres estructurales en el proceso marginal de esta variable. Puesto que estas modificaciones no afectaron los parámetros del modelo del PIB, se puede concluir que el tipo de cambio real es una variable superexógena para los mismos y, por ello, la causalidad inversa no puede aplicar.

\section{V.2 Origen del Efecto de Largo Plazo de la Demanda Externa}

Otro aspecto importante de las ecuaciones presentadas es que la tendencia del PIB mexicano la determine el índice de la producción industrial de los Estados Unidos. Sin embargo, la fuerte integración de México con ese país no es única. Canadá es el otro socio económico principal de los Estados Unidos y su dependencia se refleja en que su PIB está casi cointegrado con el de los Estados Unidos (la correlación de largo plazo excede $0.8^{7}$ ). Si los PIBs de Canadá y los Estados Unidos estuvieran cointegrados dicha correlación de largo plazo sería igual a uno, lo cual está muy cerca de ocurrir con el valor de 0.8 mencionado. Ello indica que la tendencia de la economía canadiense está fuertemente influenciada pero no completamente determinada por la de los Estados Unidos.

Los resultados del presente trabajo se encuentran probablemente relacionados con los drásticos cambios institucionales provocados por la crisis de la deuda, con las restricciones financieras a la demanda interna que resultaron de ella y con las políticas de apertura comercial de los años ochenta. Estos eventos comienzan a gestarse aproximadamente entre 1982 y 1985 mientras que las ecuaciones lo captan desde 1980. Más adelante se discute porque estas ecuaciones funcionan de esta manera en el período de referencia.

\footnotetext{
${ }^{7}$ La correlación de largo plazo es la función de coherencia a frecuencia cero (ver Brockwell y Davis 1991). Este resultado es robusto a través de diferentes submuestras. Se utilizaron los períodos 1957-2000 y 1980-2000 con resultados similares. Se usó tanto la función integrada a S-plus y el programa escrito por Albuquerque (2001) que calcula la correlación de largo plazo a frecuencia cero por un método no paramétrico.
} 
De acuerdo a Lustig (1998), antes de la crisis de 1982 el gobierno controlaba directa o indirectamente entre 60 y $80 \%$ de la economía. El costo de esta intervención se reflejó en el fuerte aumento de la deuda externa que detonó la crisis. Como consecuencia de ella, el sector público redujo su tamaño e influencia renunciando a la propiedad de empresas, reduciendo gastos y subsidios y eliminando controles de precios. Después de estos eventos, la demanda de la economía estadounidense, principalmente la proveniente de su sector industrial, se convirtió en la principal fuerza motriz de la economía mexicana. El aprovechamiento de la demanda externa fue posible gracias al abandono de décadas de políticas proteccionistas y la apertura de la economía con la afiliación del país al GATT en 1985.

Durante el período de muestra, México creció en promedio casi lo mismo que la economía estadounidense $^{8}$ porque un impulso de crecimiento interno ha estado ausente. Antes de la crisis, el gasto gubernamental tenía un papel fundamental. Este se financiaba en parte con la deuda externa que eventualmente dejó al país con una gran carga económica. Sachs (1989) trató de explicar la baja tasa de crecimiento de la economía mexicana con un argumento basado en la carga que legó la deuda externa. Este autor propuso que el temor de confiscación a través de impuestos u otras políticas para pagar la deuda hacía a los inversionistas excesivamente precavidos. De alguna manera, el miedo derivado de la fragilidad financiera del sector público podría estar aún presente y por ello la inversión privada se condiciona fundamentalmente a la evolución de la economía de los Estados Unidos. Bergoeing et al. (2001) hallaron la hipótesis de Sachs "congruente" para el caso mexicano aunque no para el chileno.

\section{V.3 Análisis Para Datos Previos a 1980}

Las ecuaciones que se presentaron en este artículo se estiman desde 1980. Sin embargo, una fecha más tardía (por ejemplo 1983) hace que la evidencia de cointegración se vuelva aún más robusta y que el ajuste mejore. De hecho, en la Gráfica 5 se muestra que el ajuste al principio de la muestra no es tan bueno. Esto sugiere que quizás un cambio estructural

\footnotetext{
${ }^{8}$ De 1980 a 2000 la tasa trimestral de crecimiento promedio de la producción industrial de los Estados Unidos fue de $0.7 \%$ mientras que la del PIB mexicano resultó de $0.69 \%$.
} 
ocurrió en esas fechas pero éste no es fácil de detectar con la información disponible. Si los datos de principios de los ochenta no se comportan de manera radicalmente diferente, entonces la estimación y la evaluación no van a detectar problemas. A continuación se discute como se abordó este problema.

Una hipótesis de este artículo es que las relaciones encontradas reflejan un fenómeno que se gestó en los años ochenta. Para probarla es necesario mirar hacia atrás. Datos de cuentas nacionales con frecuencia trimestral para México no están disponibles antes de 1980 pero sí se pueden utilizar datos anuales del PIB desde mucho antes y datos mensuales del índice de la producción industrial desde 1970.

No es posible estimar una relación como la ecuación 1 con datos anuales para toda la muestra disponible y la razón se ve claramente en el primer panel de la Gráfica 6, donde se presenta el logaritmo del cociente del PIB mexicano al PIB estadounidense para el período 1945-2000 para obtener una perspectiva del crecimiento relativo entre ambos países.

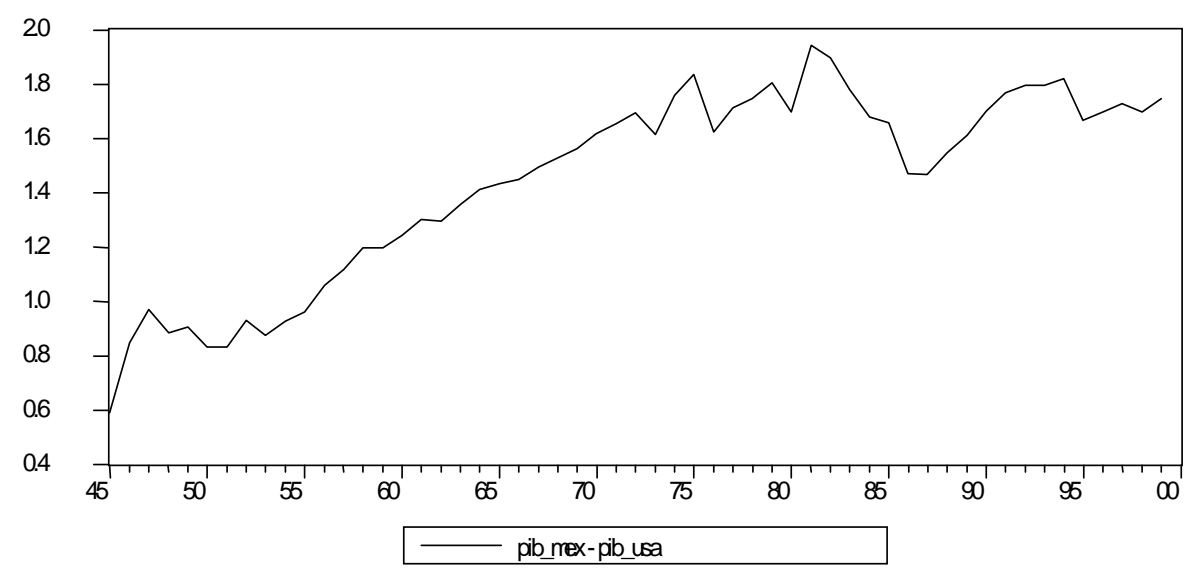

Se puede observar que la línea tiene una tendencia positiva bien definida hasta 1981. Esta proviene del hecho de que la economía mexicana crecía considerablemente más rápido que la de los Estados Unidos. Desde la mitad de los setenta se observan altibajos que no ocurrían previamente. A partir de 1982 la línea de crecimiento relativo se estabiliza a un nivel constante aunque con fluctuaciones aún más pronunciadas (relacionadas con las del tipo de cambio real), lo cual se puede interpretar a partir de los resultados del artículo: ambas economías crecieron en promedio al mismo ritmo desde los ochenta. 
Evidentemente, la ecuación 1 del PIB no aplica para la primera parte de la muestra y lo mismo debe suceder con las ecuaciones de los componentes.

Las fluctuaciones observadas a finales de los setenta están asociadas con los efectos de las devaluaciones de 1976 y 1977. De hecho, se podría pensar que la ecuación 1 podría funcionar desde esta época, al menos en cuanto al efecto del tipo de cambio real. Para explorar esta posibilidad se utilizó el índice mensual de la producción industrial de México como una variable aproximada de la actividad económica global en lugar del PIB. Se estimaron varias ecuaciones para dicho índice, condicionando en las mismas variables que la ecuación 1. Para el período 1970-1981, los coeficientes no fueron significativamente diferentes de cero. Para el período 1970-2000 se pueden obtener coeficientes de cointegración válidos únicamente al $10 \%$ de significación y los modelos de corrección de error asociados son inestables. Ambos resultados indican que los parámetros que se pueden estimar para el período 1970-1981 tienen un elevado error estándar, lo que permite que los estimados para el resto de la muestra no sean rechazados estadísticamente. Esto también explica porque las ecuaciones se pueden obtener desde 1980, aun cuando los eventos que parecen explicarlas ocurrieron después del inicio de la muestra de estimación. En contraste, a partir de 1983 los parámetros estimados para submuestras de razonable tamaño resultan muy similares y con errores estándar pequeños.

\section{Conclusiones}

La integración de las economías de México y los Estados Unidos es un proceso muy avanzado. Este comenzó a intensificarse desde principios de los años ochenta como una respuesta de la economía nacional para salir de la crisis de ese período. Desde entonces, la actividad económica de los Estados Unidos ha determinado la tendencia de la economía mexicana mientras que las fluctuaciones alrededor de la misma han estado relacionadas con los altibajos del tipo de cambio real.

En este artículo se muestra que el PIB y sus componentes tienen relaciones de cointegración con el índice de la producción industrial de los Estados Unidos y con el tipo de cambio real. Los modelos de corrección de error son bien comportados estadísticamente. 
Los resultados implican un elevado grado de integración entre las dos economías que se gestó desde principios de los ochenta y que no existía previamente. También dan una perspectiva nueva al conocido problema empírico del impacto del tipo de cambio real sobre la actividad económica de un país en desarrollo: a pesar del fuerte efecto positivo de una devaluación sobre la balanza comercial, el conjunto de la economía no responde de la misma manera. La detección de este efecto no es nueva en la literatura pero su presencia en una relación de largo plazo sí. 


\section{Referencias}

Albuquerque, Pedro H. October 2001.A Simple Nonparametric Long-run Correlation Estimator with an Application to Latin American Stock Returns, Mimeo, University de Wisconsin.

Bergoeing, R., P. J. Kehoe, T. J. Kehoe y R. Soto (2002a) “A Decade Lost and Found: Mexico and Chile in the 1980s," Review of Economic Dynamics 5, 166-205.

Bergoeing, R., P. J. Kehoe, T. J. Kehoe y R. Soto (2002b) 'Policy-Driven Productivity in Chile and Mexico in the 1980s and 1990s," NBER Working Papers Series, WP 8892.

Brockwell, P. J. and Richard A. Davis (1991) Time Series and Methods, 2nd. edition. Springer-Verlag.

Buffie, E., "Mexico 1958-1986: From Stabilizing Development to Debt Crisis," en Jeffrey Sachs, ed. Developing Country and the World Economy, University of Chicago Press, 1989.

Campos, J. and N. R. Ericsson (2000) “Constructive data mining: Modelling consumer's expenditure in Venezuela," Board of Governors of the Federal Reserve System, International Finance Discussion Papers, Abril.

De Brouwer, Gordon y Neil R. Ericsson (1998) “Modelling Inflation in Australia," Journal of Business \& Economic Statistics, Octubre 1998, Vol. 16, No. 4.

Engle, Robert, David F. Hendry (1993) 'Testing Super Exogeneity and Invariance in Regression Models," Journal of Econometrics, 56, 119-139.

Ericsson, Neil R. and James G. MacKinnon, “Distributions of Error Correction Tests for Cointegration," International Finance Discussion Paper No. 655, Board of Governors of the Federal Reserve System, 1999. 
Ericsson, Neil R., John S. Irons and Ralph W. Tryon (2001) "Output and Inflation in the Long Run," Journal of Applied Econometrics, 16: 241-253.

Granger, C.W.J. y Timo Teräsvirta (1993) Modelling Nonlinear Economic Relationships, Oxford University Press.

Hendry, D. F. y Neil R. Ericsson (1991) “'Modelling the demand for narrow money in the United Kingdom and the United States," European Economic Review, 35, 833-886, NorthHolland. Hoover, K. D. y S. J. Perez (1999) “Data Mining Reconsidered: Encompassing and the General to Specific Search," Econometrics Journal, 2, 2, 167-191 (with discussion).

Johansen, S. y K. Juselius (1992) "Testing Structural Hypothesis in a Multivariate Cointegration Analysis of the PPP and UIP for the UK," Journal of Econometrics, 53, 211244.

Juselius, Katarina (2001) “European Integration and Monetary Transmission Mechanisms:

The Case of Italy," Journal of Applied Econometrics, 16, 341-358.

Kamin, Steve B. and John H. Rogers (2000) Output and the real exchange in developing countries: an application to Mexico, Journal of Development Economics Vol. 61, 85-109.

Lustig, N. (1998) Mexico: The Remaking of an Economy. Second Edition. Washington D. C., Brookings Institution.

Maddala G. S., In-Moo Kim (1998) "Unit Roots, Cointegration and Structural Change", Cambridge University Press.

Sachs, J. (1989), “The Debt Overhang of Developing Countries,” en R. Findlay, G. Calvo,

P. Kouri and J. Braga de Macedo, editors, Debt, Stabilization and Development: Essays in Memory of Carlos Diaz-Alejandro. Oxford: Blackwell, 80-102. 
Senhadji, A. y C. Montenegro (1998), “Time Series Analysis of Export Demand Equations: A Cross-Country Analysis", IMF Working Paper, 98/149, October. 